\title{
The Duals of the 2-Modular Irreducible Modules of the Alternating Groups
}

John MURRAY

Department of Mathematics $\mathcal{E}$ Statistics, Maynooth University, Co. Kildare, Ireland

E-mail: John.Murray@mu.ie

Received January 04, 2018, in final form April 04, 2018; Published online April 17, 2018

https://doi.org/10.3842/SIGMA.2018.033

Abstract. We determine the dual modules of all irreducible modules of alternating groups over fields of characteristic 2 .

Key words: symmetric group; alternating group; dual module; irreducible module; characteristic 2

2010 Mathematics Subject Classification: 20C30

\section{Introduction and statement of the result}

Let $\mathcal{S}_{n}$ be the symmetric group of degree $n \geq 1$ and let $k$ be a field of characteristic $p>0$. In [7, Theorem 11.5] G. James constructed all irreducible $k \mathcal{S}_{n}$-modules $D^{\lambda}$ where $\lambda$ ranges over the $p$-regular partitions of $n$. Here a partition is $p$-regular if each of its parts occurs with multiplicity less than $p$.

As the alternating group $\mathcal{A}_{n}$ has index 2 in $\mathcal{S}_{n}$, the restriction $D^{\lambda} \downarrow_{\mathcal{A}_{n}}$ is either irreducible or splits as a direct sum of two non-isomorphic irreducible $k \mathcal{A}_{n}$-modules. Moreover, every irreducible $k \mathcal{A}_{n}$-module is a direct summand of some $D^{\lambda} \downarrow_{\mathcal{A}_{n}}$.

Henceforth we will assume, unless stated otherwise, that $k$ is a field of characteristic 2 which is a splitting field for the alternating group $\mathcal{A}_{n}$. For this, it suffices that $k$ contains the finite field $\mathbb{F}_{4}$. D. Benson [1] has classified all irreducible $k \mathcal{A}_{n}$-modules:

Proposition 1.1. Let $\lambda=\left(\lambda_{1}>\lambda_{2}>\cdots>\lambda_{2 s-1}>\lambda_{2 s} \geq 0\right)$ be a strict partition of $n$. Then $D^{\lambda} \downarrow_{\mathcal{A}_{n}}$ is reducible if and only if

(i) $\lambda_{2 j-1}-\lambda_{2 j}=1$ or 2 , for $j=1, \ldots, s$, and

(ii) $\lambda_{2 j-1}+\lambda_{2 j} \not \equiv 2(\bmod 4)$, for $j=1, \ldots, s$.

In this note we determine the dual of each irreducible $k \mathcal{A}_{n}$-module. Now $D^{\lambda} \downarrow \mathcal{A}_{n}$ is a self-dual $k \mathcal{A}_{n}$-module, as $D^{\lambda}$ is a self-dual $k \mathcal{S}_{n}$-module. So we only need to determine the dual of an irreducible $k \mathcal{A}_{n}$-module which is a direct summand of $D^{\lambda} \downarrow_{\mathcal{A}_{n}}$, when this module is reducible.

Theorem 1.2. Let $\lambda$ be a strict partition of $n$ such that $D^{\lambda} \downarrow_{\mathcal{A}_{n}}$ is reducible. Then the two irreducible direct summands of $D^{\lambda} \downarrow_{\mathcal{A}_{n}}$ are self-dual if $\sum_{j=1}^{s} \lambda_{2 j}$ is even and are dual to each other if $\sum_{j=1}^{s} \lambda_{2 j}$ is odd.

This paper is a contribution to the Special Issue on the Representation Theory of the Symmetric Groups and Related Topics. The full collection is available at https://www.emis.de/journals/SIGMA/symmetric-groups2018.html 
For example $D^{(7,5,1)} \downarrow_{\mathcal{A}_{13}} \cong S \oplus S^{*}$, for a non self-dual irreducible $k \mathcal{A}_{13}$-module $S$, and $D^{(5,4,3,1)} \downarrow_{\mathcal{A}_{13}}$ decomposes similarly. On the other hand $D^{(7,6)} \downarrow_{\mathcal{A}_{13}} \cong S_{1} \oplus S_{2}$ where $S_{1}$ and $S_{2}$ are irreducible and self-dual.

In order to prove Theorem 1.2, we use the following elementary result, which requires the assumption that $k$ has characteristic 2 :

Lemma 1.3. Let $G$ be a finite group and let $M$ be a semisimple $k G$-module which affords a non-degenerate $G$-invariant symmetric bilinear form $B$. Suppose that $B(t m, m) \neq 0$, for some involution $t \in G$ and some $m \in M$. Then $M$ has a self-dual irreducible direct summand.

Proof. We have $M=\bigoplus_{i=1}^{n} M_{i}$, for some $n \geq 1$ and irreducible $k G$-modules $M_{1}, \ldots, M_{n}$. Write $m=\sum m_{i}$, with $m_{i} \in M_{i}$, for all $i$. Then

$$
\begin{aligned}
B(t m, m) & =\sum_{1 \leq i \leq n} B\left(t m_{i}, m_{i}\right)+\sum_{1 \leq i<j \leq n}^{n}\left(B\left(t m_{i}, m_{j}\right)+B\left(t m_{j}, m_{i}\right)\right) \\
& =\sum_{1 \leq i \leq n} B\left(t m_{i}, m_{i}\right) .
\end{aligned}
$$

The last equality follows from the fact that $\operatorname{char}(k)=2$ and

$$
B\left(t m_{i}, m_{j}\right)=B\left(m_{i}, t^{-1} m_{j}\right)=B\left(m_{i}, t m_{j}\right)=B\left(t m_{j}, m_{i}\right) .
$$

Without loss of generality $B\left(t m_{1}, m_{1}\right) \neq 0$. Then $B$ restricts to a non-zero $G$-invariant symmetric bilinear form $B_{1}$ on $M_{1}$. As $M_{1}$ is irreducible, $B_{1}$ is non-degenerate. So $M_{1}$ is isomorphic to its $k G$-dual $M_{1}^{*}$.

\section{Known results on the symmetric and alternating groups}

\subsection{The irreducible modules of the symmetric groups}

We use the ideas and notation of [7]. In particular for each partition $\lambda$ of $n$, James defines the Young diagram $[\lambda]$ of $\lambda$, and the notions of a $\lambda$-tableau and a $\lambda$-tabloid.

Fix a $\lambda$-tableau $x$. So $x$ is a filling of $[\lambda]$ with the symbols $\{1, \ldots, n\}$. The corresponding $\lambda$-tabloid is $\{x\}:=\left\{\sigma(x) \mid \sigma \in R_{x}\right\}$, where $R_{x}$ is the row stabilizer of $x$. We regard $\{x\}$ as an ordered set partition of $\{1, \ldots, n\}$. The $\mathbb{Z}$-span of the $\lambda$-tabloids forms the $\mathbb{Z} \mathcal{S}_{n}$-lattice $M^{\lambda}$, and the set of $\lambda$-tabloids is an $\mathcal{S}_{n}$-invariant $\mathbb{Z}$-basis of $M^{\lambda}$.

Recall from [7, Section 4] that corresponding to each tableau $x$ there is a polytabloid $e_{x}:=$ $\sum \operatorname{sgn}(\sigma)\{\sigma x\}$ in $M^{\lambda}$. Here $\sigma$ ranges over the permutations in the column stabilizer $C_{x}$ of the tableau $x$. The Specht lattice $S^{\lambda}$ is defined to be the $\mathbb{Z}$-span of all $\lambda$-polytabloids. In particular $S^{\lambda}$ is a $\mathbb{Z} \mathcal{S}_{n}$-sublattice of $M^{\lambda}$; it has as $\mathbb{Z}$-basis the polytabloids corresponding to the standard $\lambda$-tableaux (i.e., the numbers increase from left-to-right along rows, and from top-to-bottom along columns).

Now James defines $\langle$,$\rangle to be the symmetric bilinear form on M^{\lambda}$ which makes the tabloids into an orthonormal basis. As the tabloids are permuted by the action of $\mathcal{S}_{n}$, it is clear that $\langle$, is $\mathcal{S}_{n}$-invariant.

Suppose now that $\lambda$ is a strict partition and consider the unique permutation $\tau \in R_{x}$ which reverses the order of the symbols in each row of the tableau $x$. In [7, Lemma 10.4] James shows that $\left\langle\tau e_{x}, e_{x}\right\rangle=1$, as $\{x\}$ is the only tabloid common to $e_{x}$ and $e_{\tau x}$ (in fact James proves that $\left\langle\tau e_{x}, e_{x}\right\rangle$ is coprime to $p$, if $\lambda$ is $p$-regular, for some prime $p$ ). Set $J^{\lambda}:=\left\{x \in S^{\lambda} \mid\langle x, y\rangle \in 2 \mathbb{Z}\right.$, for all $\left.y \in S^{\lambda}\right\}$. Then $2 S^{\lambda} \subseteq J^{\lambda}$ and it follows from [7, Theorem 4.9] that $D^{\lambda}:=\left(S^{\lambda} / J^{\lambda}\right) \otimes_{\mathbb{F}_{2}} k$ is an absolutely irreducible $k \mathcal{S}_{n}$-module, for any field $k$ of characteristic 2 . 


\subsection{The real 2-regular conjugacy classes of the alternating groups}

A conjugacy class of a finite group $G$ is said to be 2-regular if its elements have odd order. $\mathrm{R}$. Brauer proved that the number of irreducible $k G$-modules equals the number of 2-regular conjugacy classes of $G$ [4]. Now Brauer's permutation lemma holds for arbitrary fields [3, footnote 19]. So it is clear that the number of self-dual irreducible $k G$-modules equals the number of real 2-regular conjugacy classes of $G$.

We review some well known facts about the 2-regular conjugacy classes of the alternating group. See for example [8, Section 2.5].

Corresponding to each partition $\mu$ of $n$ there is a conjugacy class $C_{\mu}$ of $\mathcal{S}_{n}$; its elements consist of all permutations of $n$ whose orbits on $\{1, \ldots, n\}$ have sizes $\left\{\mu_{1}, \ldots, \mu_{\ell}\right\}$ (as multiset). So $C_{\mu}$ is 2-regular if and only if each $\mu_{i}$ is odd.

Let $\mu$ be a partition of $n$ into odd parts. Then $C_{\mu} \subseteq \mathcal{A}_{n}$. If $\mu$ has repeated parts then $C_{\mu}$ is a conjugacy class of $\mathcal{A}_{n}$. As $C_{\mu}$ is closed under taking inverses, $C_{\mu}$ is a real conjugacy class of $\mathcal{A}_{n}$.

Now assume that $\mu$ has distinct parts. Then $C_{\mu}$ is a union of two conjugacy classes $C_{\mu}^{ \pm}$ of $\mathcal{A}_{n}$. Set $m:=\frac{n-\ell(\mu)}{2}$ and let $z \in C_{\mu}$. Then $z$ is inverted by an involution $t \in \mathcal{S}_{n}$ of cycle type $\left(2^{m}, 1^{n-2 m}\right)$. Since $\mathrm{C}_{\mathcal{S}_{n}}(z) \cong \prod \mathbb{Z} / \mu_{j} \mathbb{Z}$ is odd, $t$ generates a Sylow 2-subgroup of the extended centralizer $\mathrm{C}_{\mathcal{S}_{n}}^{*}(z)$ of $z$ in $\mathcal{S}_{n}$. It follows that $z$ is conjugate to $z^{-1}$ in $\mathcal{A}_{n}$ if and only if $t \in \mathcal{A}_{n}$.

This shows that $C_{\mu}^{ \pm}$are real classes of $\mathcal{A}_{n}$ if and only if $\frac{n-\ell(\mu)}{2}$ is even. This and the discussion above shows:

Lemma 2.1. The number of self-dual irreducible $k \mathcal{A}_{n}$-modules equals the number of non-strict odd partitions of $n$ plus twice the number of strict odd partitions $\mu$ of $n$ for which $\frac{n-\ell(\mu)}{2}$ is even.

\section{Bressoud's bijection}

We need a special case of a partition identity of I. Schur [9]. This was already used by Benson in his proof of Proposition 1.1:

Proposition 3.1 (Schur, 1926). The number of strict partitions of $n$ into odd parts equals the number of strict partitions of $n$ into parts congruent to $0, \pm 1(\bmod 4)$ where consecutive parts differ by at least 4 and consecutive even parts differ by at least 8.

D. Bressoud [5] has constructed a bijection between the relevant sets of partitions. We describe a simplified version of this bijection.

Let $\mu=\left(\mu_{1}>\mu_{2}>\cdots>\mu_{\ell}\right)$ be a strict partition of $n$ whose parts are all odd. We subdivide $\mu$ into 'blocks' of at most two parts, working recursively from largest to smallest parts. Let $j \geq 1$ and suppose that $\mu_{1}, \mu_{2}, \ldots, \mu_{j-1}$ have already been assigned to blocks. We form the block $\left\{\mu_{j}, \mu_{j+1}\right\}$ if $\mu_{j}=\mu_{j+1}+2$, and the block $\left\{\mu_{j}\right\}$ otherwise (if $\left.\mu_{j} \geq \mu_{j+1}+4\right)$. Let $s$ be the number of resulting blocks of $\mu$.

Next we form the sequence of positive integers $\sigma=\left(\sigma_{1}, \sigma_{2}, \ldots, \sigma_{s}\right)$, where $\sigma_{j}$ is the sum of the parts in the $j$-th block of $\mu$. Then the $\sigma_{j}$ are distinct, as the odd parts form a decreasing sequence, with minimal difference 4 , and the even parts form a decreasing sequence, with minimal difference 8. Moreover, each even $\sigma_{j}$ is the sum of a pair of consecutive odd integers. So $\sigma_{j} \not \equiv 2$ $(\bmod 4)$, for all $j>0$.

We get a composition $\zeta$ of $n+2 s(s-1)$ by defining

$$
\zeta_{1}=\sigma_{1}, \zeta_{2}=\sigma_{2}+4, \ldots, \zeta_{s}=\sigma_{s}+4(s-1) .
$$

The even $\zeta_{j}$ form a decreasing sequence, with minimal difference 4 , and the odd $\zeta_{j}$ form a weakly decreasing sequence $\left(\zeta_{j}=\zeta_{j+1}\right.$ if and only if $\zeta_{j}, \zeta_{j+1}$ represent two singleton blocks $\{2 k-1\}$ and $\{2 k-5\}$ of $\mu$, for some $k \geq 0)$. 
Choose a permutation $\tau$ such that $\zeta_{\tau 1} \geq \zeta_{\tau 2} \geq \cdots \geq \zeta_{\tau s}$. Then we get a strict partition $\gamma$ of $n$ by defining

$$
\gamma_{1}=\zeta_{\tau 1}, \gamma_{2}=\zeta_{\tau 2}-4, \ldots, \gamma_{s}=\zeta_{\tau s}-4(s-1) .
$$

By construction, the minimal difference between the parts of $\gamma$ is 4 and the minimal difference between the even parts of $\gamma$ is 8 . Moreover, $\gamma_{j} \equiv \zeta_{\tau j}(\bmod 4)$. So $\gamma_{j} \not \equiv 2(\bmod 4)$. Then $\mu \rightarrow \gamma$ is Bressoud's bijection.

Finally form a strict partition $\lambda$ of $n$ which has $2 s-1$ or $2 s$ parts, by defining

$$
\left(\lambda_{2 j-1}, \lambda_{2 j}\right)= \begin{cases}\left(\frac{\gamma_{j}}{2}+1, \frac{\gamma_{j}}{2}-1\right), & \text { if } \gamma_{j} \text { is even or } \\ \left(\frac{\gamma_{j}+1}{2}, \frac{\gamma_{j}-1}{2}\right), & \text { if } \gamma_{j} \text { is odd. }\end{cases}
$$

Then $\lambda$ satisfies the constraints (i) and (ii) of Proposition 1.1. Conversely, it is easy to see that if $\lambda$ satisfies these constraints, then $\lambda$ is the image of some strict odd partition $\mu$ of $n$ under the above sequence of operations.

Lemma 3.2. Let $\mu$ be a strict-odd partition of $n$ and let $\lambda$ be the strict partition of $n$ constructed from $\mu$ as above. Then $\frac{n-\ell(\mu)}{2}=\sum \lambda_{2 j}$.

Proof. Each pair of consecutive parts $\lambda_{2 j-1}, \lambda_{2 j}$ of $\lambda$ corresponds to a block $\mathcal{B}$ of $\mu$. Moreover by our description of Bressoud's bijection, there are integers $q_{1}, \ldots, q_{s}$, with $\sum q_{j}=0$ such that

$$
\left(\lambda_{2 j-1}+2 q_{j}, \lambda_{2 j}+2 q_{j}\right)= \begin{cases}\left(\frac{\mu_{i}+1}{2}, \frac{\mu_{i}-1}{2}\right), & \text { if } \mathcal{B}=\left\{\mu_{i}\right\}, \\ \left(\mu_{i}, \mu_{i+1}\right), & \text { if } \mathcal{B}=\left\{\mu_{i}, \mu_{i+1}\right\} .\end{cases}
$$

In case $\mathcal{B}=\left\{\mu_{i}, \mu_{i+1}\right\}$, we have $\mu_{i}=\mu_{i+1}+2$ and thus $\frac{\mu_{i}-1}{2}+\frac{\mu_{i+1}-1}{2}=\lambda_{2 j}+2 q_{j}$. We conclude that

$$
\frac{n-\ell(\mu)}{2}=\sum_{i=1}^{\ell(\mu)} \frac{\mu_{i}-1}{2}=\sum_{j=1}^{s}\left(\lambda_{2 j}+2 q_{j}\right)=\sum_{j=1}^{s} \lambda_{2 j} .
$$

\section{Proof of Theorem 1.2}

Let $\mathrm{D}(n)$ be the set of strict partitions of $n$ and let $\mathrm{S}(n)$ be the set of strict partitions of $n$ which satisfy conditions (i) and (ii) in Proposition 1.1. So there are $2|\mathrm{~S}(n)|+|\mathrm{D}(n) \backslash \mathrm{S}(n)|$ irreducible $k \mathcal{A}_{n}$-modules.

Next set $\mathrm{S}(n)^{+}:=\left\{\lambda \in \mathrm{S}(n) \mid \sum \lambda_{2 j}\right.$ is even $\}$. Then it follows from Lemmas 2.1 and 3.2 that the number of self-dual irreducible $k \mathcal{A}_{n}$-modules equals $2\left|\mathrm{~S}(n)^{+}\right|+|\mathrm{D}(n) \backslash \mathrm{S}(n)|$. Now $D^{\lambda} \downarrow_{\mathcal{A}_{n}}$ is an irreducible self-dual $k \mathcal{A}_{n}$-module, for $\lambda \in \mathrm{D}(n) \backslash \mathrm{S}(n)$. So we can prove Theorem 1.2 by showing that the irreducible direct summands of $D^{\lambda} \downarrow_{\mathcal{A}_{n}}$ are self-dual for all $\lambda \in \mathrm{S}(n)^{+}$.

Suppose then that $\lambda \in \mathrm{S}(n)^{+}$. Let $\tau \in \mathcal{S}_{n}$ be the permutation which reverses each row of a $\lambda$-tableau, as discussed in Section 2.1. We claim that $\tau \in \mathcal{A}_{n}$. For $\tau$ is a product of $\sum_{i=1}^{2 s}\left\lfloor\frac{\lambda_{j}}{2}\right\rfloor$ commuting transpositions. Now $\left\lfloor\frac{\lambda_{2 j-1}}{2}\right\rfloor+\left\lfloor\frac{\lambda_{2 j}}{2}\right\rfloor=\lambda_{2 j}$, as $\lambda_{2 j-1}-\lambda_{2 j}=1$, or $\lambda_{2 j-1}-\lambda_{2 j}=2$ and both $\lambda_{2 j-1}$ and $\lambda_{2 j}$ are odd. So $\sum_{i=1}^{2 s}\left\lfloor\frac{\lambda_{i}}{2}\right\rfloor=\sum_{j=1}^{s} \lambda_{2 j}$ is even. This proves the claim.

Since $D^{\lambda}$ is irreducible and the form $\langle$,$\rangle is non-zero, \langle$,$\rangle is non-degenerate on D^{\lambda}$. Write $D^{\lambda} \downarrow_{\mathcal{A}_{n}}=S_{1} \oplus S_{2}$, where $S_{1}$ and $S_{2}$ are non-isomorphic irreducible modules. As $\tau \in \mathcal{A}_{n}$, it follows from Lemma 1.3 that we may assume that $S_{1}$ is self-dual. Now $S_{2}^{*} ¥ S_{1}^{*} \cong S_{1}$ and $S_{2}^{*}$ is isomorphic to a direct summand of $D^{\lambda} \downarrow \mathcal{A}_{n}$. So $S_{2}$ is also self-dual. This completes the proof of the theorem. 


\section{$5 \quad$ Irreducible modules of alternating groups over fields of odd characteristic}

We now comment briefly on what happens when $k$ is a splitting field for $\mathcal{A}_{n}$ which has odd characteristic $p$. Let sgn be the sign representation of $k \mathcal{S}_{n}$. So sgn is 1-dimensional but nontrivial. G. Mullineux defined a bijection $\lambda \rightarrow \lambda^{M}$ on the $p$-regular partitions of $n$ and conjectured that $D^{\lambda} \otimes \operatorname{sgn}=D^{\lambda^{M}}$ for all $p$-regular partitions $\lambda$ of $n$. This was only proved in the 1990's by Kleshchev and Ford-Kleshchev. See [6] for details.

Now $D^{\lambda} \downarrow_{\mathcal{A}_{n}} \cong D^{\lambda^{M}} \downarrow_{\mathcal{A}_{n}}$, and $D^{\lambda} \downarrow_{\mathcal{A}_{n}}$ is irreducible if and only if $\lambda \neq \lambda^{M}$ See [2] for details. Moreover $D^{\lambda}$ and $D^{\lambda^{M}}$ are duals of each other, by [7, Theorem 6.6]. So $D^{\lambda} \downarrow_{\mathcal{A}_{n}}$ is self-dual, if $\lambda \neq \lambda^{M}$. However when $\lambda=\lambda^{M}$, we do not know how to determine when the two irreducible direct summands of $D^{\lambda} \downarrow_{\mathcal{A}_{n}}$ are self-dual.

\section{Acknowledgement}

D. Benson told me that it was an open problem to determine the self-dual irreducible $k \mathcal{A}_{n^{-}}$ modules. G.E. Andrews directed me to Bressoud's paper [5]. We also thank the anonymous referees for their comments, which helped to improve the clarity of this paper.

\section{References}

[1] Benson D., Spin modules for symmetric groups, J. London Math. Soc. 38 (1988), 250-262.

[2] Bessenrodt C., On the representation theory of alternating groups, Algebra Colloq. 10 (2003), 241-250.

[3] Brauer R., On the connection between the ordinary and the modular characters of groups of finite order, Ann. of Math. 42 (1941), 926-935.

[4] Brauer R., Nesbitt C., On the modular characters of groups, Ann. of Math. 42 (1941), 556-590.

[5] Bressoud D.M., A combinatorial proof of Schur's 1926 partition theorem, Proc. Amer. Math. Soc. 79 (1980), 338-340.

[6] Ford B., Kleshchev A.S., A proof of the Mullineux conjecture, Math. Z. 226 (1997), 267-308.

[7] James G.D., The representation theory of the symmetric groups, Lecture Notes in Math., Vol. 682, Springer, Berlin, 1978.

[8] James G.D., Kerber A., The representation theory of the symmetric group, Encyclopedia of Mathematics and its Applications, Vol. 16, Addison-Wesley Publishing Co., Reading, Mass., 1981.

[9] Schur I., Zur additiven Zahlentheorie, Sitzungsber. Preuß. Akad. Wiss. Phys.-Math. Kl. (1926), 488-495, Reprinted in Schur I., Gesammelte Abhandlungen, Band III, Springer-Verlag, Berlin - New York, 1973, $43-50$. 